\title{
NOVEL EFFECTS ASSOCIATED WITH THE USE OF PROBIOTICS, SYNBIOTICS AND PREBIOTICS
}

\author{
Wasim Ahmad \\ Assistant Professor \\ Department of Allied Health Sciences \\ SHS, Peshawar-KPK
}

To maintain the wellbeing of the human gut, the role of its microflora and their metabolites is fundamental. Probiotics is a Greek term, which is used to define non-living microbes that are beneficial in nature and exert numerous advantages on the host body. The intestinal microflora's function and activity may also be improved by administering some of the prebiotics (indigestible dietary fibers) independently or in a c combination with probiotics. The term "Synbiotics" is used to denote the combination of both prebiotics and probiotics. It should always be noted that numerous disorders are being associated with the abnormal function of microbiota/microflora that can be regulated and stabilized with the help of ingestion of probiotics, prebiotics or their combination. Multiple benefits are associated with the use of these probiotics, synbiotics and prebiotics including healthier digestive system, proper immune response of the gut and treatment of numerous GI tract disorders. All these issues are encountered because of treatments with new synthetic drugs that affect the digestive system as well as numerous organs in the body. Ulcerative colitis is a term that refers to chronic inflammation of the colon emerging as a result of improper immune response to intestinal microflora of the host. Researchers have studied the effects of synbiotics to treat this chronic condition ${ }^{1}$. As for the action mechanism of the synbiotics is concerned, it is assumed that they modulate the inflammatory response through expression of the cytokines, probiotic bacteria assisted modulation of the immune cells and prebiotics assisted production of certain short chain fatty acids. Jayanama and Theou studied the consequences of improper ingestion of probiotics and prebiotics in elderly patients. They revealed that physical inactivity, sarcopenia, and polypharmacy could be treated with the help of probiotics, prebiotics or a combination of both $^{2}$. All the aforementioned disorders can lead to gut dysbiosis.

The researchers have also revealed that the gut microbiota homeostasis can be improved with the help of probiotics and prebiotics and they can be a helping hand in the prevention of aging and frailty. It is evident that left and right prescriptions of antibiotics and their use caused antibiotic resistance in the pathogens. This issue can be best resolved with the ingestion of probiotics and prebiotics. The systematic evidence to treat infected chronic wounds has been provided by Brognara et al $^{3}$. Similarly, chronic wounds and burns treatment is done widely with the use of Lactobacillus plantarum. Some other researchers have reviewed the available data associated with the use of numerous prebiotics in the treatment of idiopathic constipation, and highly prevalent GIT disorrderrs ${ }^{4-6}$. This is due to the capability of prebiotics to improve the consistency of the stool, bloating and number of bowel movements. A limited data is available regarding the efficacy of prebiotics in the treatment of ulcerative colitis and pouchitis. It is concluded that probiotics, prebiotics and synbiotics have been imparting a role in treating GIT disorders including indigestion, traveler's diarrhea, IBD etc. Scientific reports have revealed their role in the treatment of cancerous disease, obesity, kidney and brain disorders. Many tools for the research of probiotics, prebiotics and synbiotics are now available; therefore, the future bio-therapeutics are going to be changed with the passage of time.

\section{REFERENCES:}

1. Roselli M, Finamore A. Use of synbiotics for ulcerative colitis treatment. Curr Clin Pharmacol. 2020;15(3):174-82.

2. Jayanama K, Theou O. Effects of probiotics and prebiotics on frailty and ageing: a narrative review. Curr Clin Pharmacol. 2020;15(3):183-92. 


\section{JGMDS}

3. Brognara L, Salmaso L, Mazzotti A, Di Martino A, Faldini C, Cauli O. Effects of probiotics in the management of infected chronic wounds: from cell culture to human studies. Curr Clin Pharmacol. 2020;15(3):193-206.

4. Naseer M, Poola S, Uraz S, Tahan V. Therapeutic effects of prebiotics in constipation: a review. Curr Clin Pharmacol. 2020;15(3):207-15.

5. Naseer M, Poola S, Ali S, Samiullah S, Tahan V. Prebiotics and probiotics in Inflammatory Bowel Disease (IBD): where are we now and where are we going?. Curr Clin Pharmacol. 2020;15(3):216-33.

6. Vlachou E, Ntikoudi A, Govina O, Lavdaniti M, Kotsalas N, Tsartsalis A, et al. Effects of probiotics on diabetic nephropathy: a systematic review. Curr Clin Pharmacol. 2020;15(3):234-42. 